\title{
PARÁMETROS DE ESTÉTICA FACIAL Y GRADIENTE SOCIOGENÉTICO EN NIÑOS CHILENOS
}

\author{
Facial esthetics and sociogenetic gradient parameters \\ in chilean children
}

Hernán M. Palomino ${ }^{(1)}$, Constanza Guzmán ${ }^{(2)}$, Soledad Urzúa ${ }^{(3)}$, Paula Aranda ${ }^{(4)}$, Pía Villanueva ${ }^{(5)}$

\begin{abstract}
RESUMEN
Objetivo: determinar valores antropométricos de estética facial de niños de 5 y 8 años de edad, con características físicas y oclusales normales, pertenecientes a diferentes grupos sociogenéticos de Santiago de Chile, debido a que es un país con marcada mezcla indígena, y compararlos entre sí según sexo y estrato para cada grupo etáreo y con los valores norteamericanos utilizados internacionalmente publicados por Farkas. Metodos: se realizó un análisis facial basado en Arnett y Farkas, utilizando fotografía digital apoyada por examen clínico, en cuatro muestras, clasificadas según dentición y estrato. Resultados: no existen diferencias entre sexo ni estrato en Chile, ni tampoco cuando estas son comparadas con las medidas norteamericanas. Conclusiones: las mediciones antropométricas faciales determinadas para los distintos grupos sociogenéticos en Santiago Chile no difieren entre si ni con las publicadas por Farkas, por lo que estas pueden ser utilizadas en la clínica en Santiago con poblaciones étnicamente mixtas.
\end{abstract}

PALABRAS CLAVES: Grupos Étnicos; Estética; Antropometría

\section{INTRODUCCIÓN}

Desde los inicios de la ortodoncia, la valoración clínica del perfil ha sido de gran importancia en el diagnóstico y tratamiento de las anomalías dentomaxilares ${ }^{1}$. Para ello, la teleradiografía y su análisis cefalométrico ha sido de gran utilidad para la determinación de los estándares de normalidad en cuanto a la forma del cráneo y al patrón facial. De esta manera se han realizado estudios en distintas poblaciones, generándose patrones con los promedios obtenidos, para cada grupo racial ${ }^{2,3}$. Sin

(1) Magíster en Odontología, Profesor Asistente Universidad de Chile, Profesor Titular Universidad Andrés Bello, Santiago, Chile; Especialista en Ortodoncia por la Universidad de Chile.

(2) Cirujano Dentista y Licenciada en Odontología por la Universidad de Chile.

(3) Cirujano Dentista, Profesor Asistente Universidad de Chile; Licenciada en Odontología y Especialista en Ortodoncia por la Universidad de Chile.

(4) Cirujano Dentista y Licenciada en Odontología por la Universidad de Chile.

(5) Fonoaudióloga, Profesor Asistente Universidad de Chile; Magíster en Ciencias Odontológicas por la Universidad de Chile. embargo, la utilización de la cefalometría como único parámetro puede producir problemas estéticos. Se deben considerar además la disposición de los tejidos blandos, ya que ellos determinan finalmente la estética facial ${ }^{4}$.

El concepto actual de estética establece que no existe una total correspondencia entre la apariencia física y el complejo óseo subyacente, por lo que deben estudiarse en forma aislada ${ }^{1,4,5}$. Es por esto que un análisis del perfil de tejidos blandos faciales y su comparación con medidas estándares de éstos, son necesarias en todas las especialidades relacionadas con cambios en rasgos faciales ${ }^{6}$, como son la cirugía ortognática, la ortodoncia, y mas recientemente la fonoaudiología, donde en la actualidad se han publicado una serie de trabajos que describen técnicas de motricidad orofacial en evaluación de estética facial y donde se describen técnicas miofuncionales y estomatognáticas para lograrla ${ }^{7-9}$.

La antropometría clínica ${ }^{10-12}$ ha resurgido, entre otros, por los estudios realizados por Farkas, quien ha realizado medidas en los tejidos blandos en los tres sentidos del espacio y en distintas edades, estableciendo valores promedios para cada una de ellas ${ }^{13-17}$. Arnett, por otra parte, presentó un análisis de diecinueve rasgos claves faciales y los analizó desde una vista frontal y lateral. En su análisis relaci- 
ona algunas mediciones con la proyección de ciertos puntos hacia una línea vertical verdadera (LVV) que pasa por el punto subnasal $4,18,19$.

De esta forma, se han planteado patrones de normalidad en cuanto a medidas faciales principalmente para la población caucásica. En nuestro medio se emplean estas medidas como estándares y, dado que la constitución genética de la población chilena actual es una mezcla de diferentes razas, cabe preguntarse si estos estándares, dados para la población caucásica, rigen para la población chilena ${ }^{1}$.

La población chilena se ha originado principalmente $(95 \%)$ de la mezcla de varones europeos (españoles) y mujeres aborígenes, llevando a la formación de una población mestiza numerosa, que desplazó en poco tiempo a la población aborigen ${ }^{20}$.

Diversos estudios han establecido la existencia una gradiente poblacional que correlaciona nivel socioeconómico con grado de mezcla indígena y europea en la ciudad de Santiago, donde los niveles socioeconómicos más altos poseen en su composición genética alrededor de un $18 \%$ de genes indígenas, mientras las poblaciones de nivel más bajo poseen un mayor porcentaje de indigenicidad de alrededor de un $37 \%{ }^{20-23}$.

Si estos sectores difieren en su constitución genética, es altamente probable que difieran también en sus medidas antropométricas faciales. El objetivo de este estudio es determinar esas medidas antropométricas, compararlas entre los distintos grupos sociogenéticos y con las medidas internacionales descritas para caucásicos por Farkas y establecer parámetros de estética facial que puedan ser aplicados a la clinica.

\section{MÉTODOS}

De una muestra total de 1059 niños de 7 diferentes colegios de Santiago, Chile, fueron seleccionados 127 sujetos de 5 y 8 años con características normales de oclusión de acuerdo a su nivel de evolución de la dentición y que se encontraran dentro del percentil 25 y 75 del índice de masa corporal ${ }^{24,25}$.

Fueron agrupados según dentición: Temporal o Mixta 1a Fase; y según estrato sociogenético: $\mathrm{SI}$ (estrato con menor índice de indigenicidad y mayor estrato socioeconómico) y SIII (estrato con mayor índice de indigenicidad y menor estrato socioeconómico) ${ }^{20-23}$.

A cada sujeto se le realizaron tres mediciones lineales con un pie de metro, según describió Farkas en su análisis antropométrico facial ${ }^{15}:$ TG'$^{\prime}-S^{\prime}$ ': Profundidad Tercio medio facial: distancia en línea recta entre punto Tragion y punto Subnasal; TG'-GN': Profundidad Tercio inferior facial: distancia en línea recta entre punto Tragion y Gnation; SN'-PN': Protrusión nasal (Columela) distancia en línea recta entre el punto subnasal y el punto pronasal (punta de la nariz).
A cada niño se le realizaron las 5 marcas faciales (puntos de $2 \mathrm{~mm}$ realizados con lápiz de tinta) descritas por Arnett ${ }^{19}$ : 1- Punto orbitario (OR'): se ubicó en el arco infraorbitario, a la altura de la pupila cuando el paciente estaba mirando de frente; 2 - Punto hueso malar (CB'): se ubicó de la siguiente manera: desde una vista lateral (3/4) se marcó la parte más prominente del malar; de frente, se ubicó en la intersección de la parte más alta del malar con una línea vertical que pasaba por el canto externo del ojo; 3- Punto base alar (AB'): se marcó en la depresión más profunda del ala de la nariz; 4- Punto subpupilar (SP'): se sitúa directamente bajo la pupila cuando el paciente mira hacia el frente. Verticalmente se ubica en la mitad de la distancia entre el punto orbitario y base alar; 5- Pogonion piel (PG'): punto más prominente y anterior del mentón óseo proyectado en tejido blando.

De cada sujeto en estudio, se tomaron fotografías del perfil en posición natural de cabeza (NHP) ${ }^{26} y$ máxima intercuspidación dentaria.

Se utilizó una plomada al lado izquierdo del paciente con la que posteriormente se determinó la línea vertical verdadera (LVV). Se traspasaron las fotografías al computador. Se utilizó el programa de dibujo Autodesk Architectural Desktop 3.3. con el cual se escalaron las fotos y se trasladó la LVV hasta hacerla coincidir con subnasal y así determinar los valores de las medidas descritas anteriormente (Figuras 1, 2, 3, 4).

Los procedimientos de recolección e interpretación de datos de esta investigación fueron aprobados por el Comité de Ética de la Escuela de Fonoaudiología mediante la resolución número: MO 03/06

Los datos se analizaron estadísticamente mediante Test $t$ de Student y de Wilcoxon. Las comparaciones se realizaron entre sexo, estratos sociogenéticos dentro de un mismo grupo etáreo y con los valores de los estudios realizados por Farkas ${ }^{27}$, obtenidos de la literatura. Las variables se agruparon en tres grupos según correspondían a tercio medio, tercio inferior o desarrollo nasal.

\section{RESULTADOS}

El la Tabla 1 se observa la totalidad de las mediciones según el analisis de Farkas y Arnett, para los cuatro grupos de la muestra. Al comparar mediante el Test de Wilcoxon las mediciones que corresponden al tercio medio, según sexo; se observa diferencia significativa en la medida AB'-LVV $(p<0.05)$, en el grupo dentición temporal $S I$, y en las medidas OR'-LVV $(p<0.01)$, CB'-LVV $(p<0.05)$, SP'$\operatorname{LVV}(p<0,05)$ y $\left.A B^{\prime}-\operatorname{LV}(p<0.05)\right)$ en el grupo de dentición mixta S III (Tabla 2).

En Tercio Inferior y Desarrollo Nasal, no se observan diferencias entre sexo para ambos estratos $y$ denticiones.

Como se aprecia en la Tabla 3, al comparar las variables obtenidas tanto en dentición temporal como 
mixta entre los estratos sociogenéticos SI y SIII, se observa que en varones en dentición temporal se presentan diferencias estadísticamente significativas en medida de desarrollo nasal SN'-PN' $(p<0,05)$; mientras que en dentición mixta se observan diferencias en las variables del tercio medio CB'-LVV y AB'LVV $(p<0,05)$, y en ambas variables del desarrollo nasal SN'-PN' y Ángulo NL $(p<0,01)$.

Al realizar la comparación en mujeres, se observaron diferencias estadísticamente significativas para las mediciones del tercio medio AB'-LVV $(p<0,01)$ en el grupo de dentición temporal, para la medida TG'$\mathrm{SN}^{\prime}(p<0,05)$ en el grupo de dentición mixta, y para la medida SN'-PN' $(p<0,01)$ en ambas denticiones.

Cabe destacar que la diferencia estadística de la medida de desarrollo nasal SN'-PN', se mantuvo constante entre los estratos sociogenéticos tanto en dentición temporal como mixta en ambos sexos. No se apreciaron diferencias para las medidas de tercio inferior. Con respecto a tercio medio, a pesar que existen diferencias, estas se presentan en variables aisladas, siendo más frecuentemente la de AB'-LVV.

Realizado el análisis de comparación entre estratos y Farkas según sexo y dentición (Tablas 4 y 5) a través de la prueba t-Student, entre los valores obtenidos por Farkas en población norteamericana ${ }^{9}$ y los valores obtenidos en nuestro estudio, según sexo y estrato, se encontraron diferencias estadísticamente significativas en: desarrollo nasal de hombres de estrato SI en dentición temporal y en estrato SIII en dentición mixta (SN'- $\left.P N^{\prime}: p<0,01\right)$, y en relación a profundidad de tercio medio en hombres de estrato SI en dentición mixta (TG'-SN': $p<0,05)$.

Con respecto a las mujeres se encontraron diferencias estadísticamente significativas en dentición temporal en relación al desarrollo nasal del estrato SI (SN'-PN': $p<0,01$ ), mientras que para el grupo en dentición mixta se encontraron diferencias en todas las variables del estrato SI y para el estrato SIII en profundidad de tercio medio y desarrollo nasal (TG'-GN' y SN'-PN': $p<0,05$ ).

Es importante recalcar que en general las mayores diferencias fueron encontradas nuevamente en relación al Desarrollo Nasal.

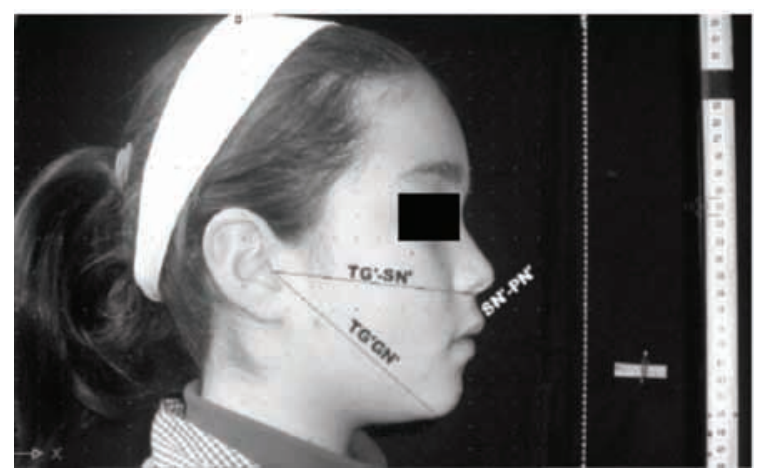

Figura 1 - Foto perfil describiendo medidas de Farkas (Análisis modificado de Farkas ${ }^{17}$ )

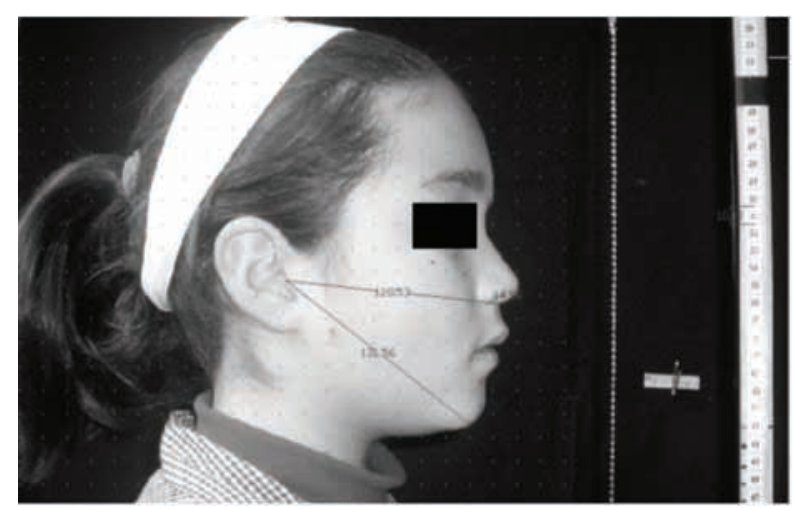

Figura 2- Foto perfil aplicando medidas de Farkas en programa computacional

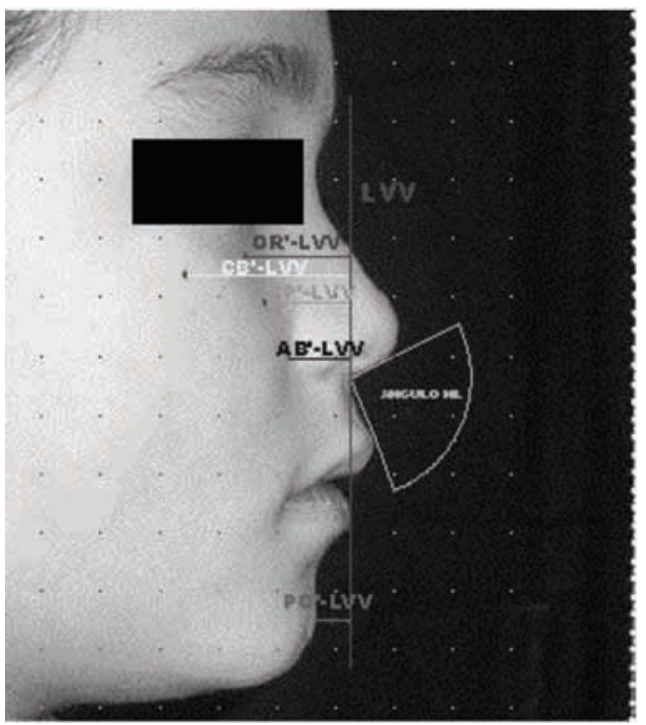

Figura 3 - Foto perfil describiendo medidas descritas por Arnett (Análisis modificado de Arnett ${ }^{19}$ )

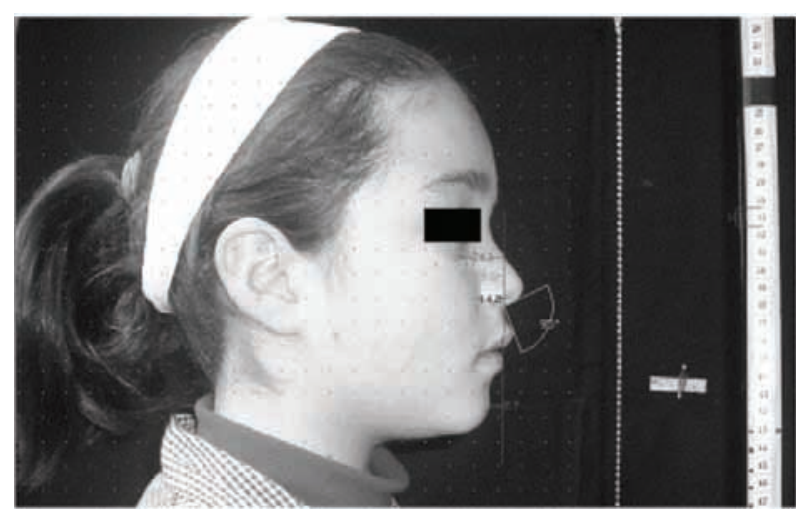

Figura 4 - Foto perfil aplicando medidas de Arnett en el programa computacional 
Tabla 1 - Tabla resumen de mediciones faciales, expresadas en milímetros ( $\mathrm{mm}$ )

\begin{tabular}{|c|c|c|c|c|c|c|c|c|c|c|c|c|c|c|c|c|c|c|c|c|c|}
\hline \multirow[b]{3}{*}{ Edad } & \multirow[b]{3}{*}{ Estrato } & \multirow[b]{3}{*}{ Sexo } & \multicolumn{11}{|c|}{ MEDIDAS TERCIO IMEDIO } & \multicolumn{4}{|c|}{ TERCIO INFERIOR } & \multicolumn{4}{|c|}{ DESARROLLO NASAL } \\
\hline & & & \multicolumn{3}{|c|}{ OR'-LVV } & \multicolumn{2}{|c|}{ CB'-LVV } & \multicolumn{2}{|c|}{ SP'-LVV } & \multicolumn{2}{|c|}{$A B^{\prime}-L V V$} & \multicolumn{2}{|c|}{ TG-SN } & \multicolumn{2}{|c|}{ PG'-LVV } & \multicolumn{2}{|c|}{ TG'-GN' } & \multicolumn{2}{|c|}{$\mathbf{S N}^{-}-\mathrm{PN}^{\prime}$} & \multicolumn{2}{|c|}{ Angulo NL } \\
\hline & & & $\mathbf{N}$ & $\bar{x}$ & DS & $\bar{x}$ & DS & $\bar{x}$ & DS & $\bar{x}$ & DS & $\bar{x}$ & DS & $\bar{x}$ & DS & $\bar{x}$ & DS & $\bar{x}$ & DS & $\bar{x}$ & $\overline{D S}$ \\
\hline \multirow{6}{*}{$\begin{array}{c}5 \\
\text { AÑOS }\end{array}$} & \multirow{2}{*}{ SI } & $\bar{M}$ & 13 & 18,71 & 4,28 & 27,04 & 5,95 & 15,98 & 3,5 & 9,58 & 2 & 106,54 & 12,15 & 9,11 & 4,87 & 116,35 & 13,02 & 14,76 & 1,28 & 106,69 & 10,07 \\
\hline & & $F$ & 14 & 16,79 & 3,59 & 24,86 & 4,2 & 14,11 & 3,32 & 7,59 & 2,14 & 101,48 & 6,67 & 7,62 & 3,64 & 111,75 & 7,21 & 14,6 & 1,27 & 110,64 & 11,01 \\
\hline & \multirow{2}{*}{ SIII } & M & 18 & 19,61 & 4,8 & 29,16 & 6,5 & 15,05 & 4,26 & 10,72 & 3,19 & 105,78 & 12,11 & 8,55 & 1,88 & 111,94 & 10,4 & 13,5 & 1,25 & 100,94 & 10,99 \\
\hline & & $F$ & 15 & 18,66 & 3,01 & 28,13 & 5,11 & 14,26 & 2,57 & \begin{tabular}{|l|}
10,4 \\
\end{tabular} & 2,06 & 105,29 & 8,47 & 9,4 & 2,02 & 111,82 & 10,09 & 12,93 & 1,33 & 104,8 & 11,03 \\
\hline & \multirow[t]{2}{*}{ Farkas } & M & 30 & - & $\cdot$ & $\cdot$ & $\cdot$ & $\cdot$ & - & - & - & 106 & 3,5 & $\cdot$ & - & 115 & $\begin{array}{c}5,3 \\
, 3\end{array}$ & 13,3 & 0,8 & 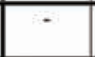 & $\cdot$ \\
\hline & & $F$ & 30 & - & - & - & - & - & - & - & - & 102,9 & 3,2 & $\cdot$ & - & 111,5 & 4,1 & 13,1 & 1,2 & - & $\cdot$ \\
\hline \multirow{6}{*}{$\begin{array}{c}8 \\
\text { ANNos }\end{array}$} & \multirow{2}{*}{ si } & $M$ & 13 & 21 & 2,97 & 32,84 & 3,57 & 16,53 & 2,43 & 11,92 & 1,65 & 105,78 & 12,11 & 10,3 & 3,88 & 111,94 & 10,4 & 13,5 & 1,25 & 111,53 & 9,93 \\
\hline & & $F$ & 8 & 19,37 & 2,55 & 29,87 & 4.05 & 14,37 & 2,77 & 10,5 & 2 & 115,75 & 5,47 & 9,62 & 2,19 & 124,88 & 8,89 & 16,5 & 1,06 & 107,5 & 11,25 \\
\hline & \multirow{2}{*}{ SIII } & $M$ & 24 & 19,75 & 2,48 & 28,7 & 4,68 & 15,12 & 2,07 & 10,7 & 1,26 & 112,5 & \begin{tabular}{|l|}
8,59 \\
\end{tabular} & 8,41 & 3,67 & 120,83 & 7,92 & 14,95 & 1,23 & 99,33 & 11,94 \\
\hline & & $F$ & 22 & 17,45 & 2,95 & 25,95 & 4,65 & 13,36 & 3,25 & \begin{tabular}{|l|}
9,63 \\
\end{tabular} & 2,21 & 108 & 9,43 & 8.4 & 2,61 & 117,5 & 9,72 & 14,81 & 1,25 & 100,04 & 11,04 \\
\hline & \multirow{2}{*}{ Farkas } & M & 51 & - & $\cdot$ & $\cdot$ & $\cdot$ & - & $\cdot$ & - & - & 111 & 3,8 & - & - & 118,6 & 4 & 15,9 & 1.3 & - & - \\
\hline & & $F$ & 51 & - & - & - & - & - & - & - & - & \begin{tabular}{|l|}
107,5 \\
\end{tabular} & 4 & $\begin{array}{l}- \\
-\end{array}$ & - & 113,9 & 4.7 & 15,5 & \begin{tabular}{|l|}
1,2 \\
\end{tabular} & - & \\
\hline
\end{tabular}

TG'-SN': Profundidad Tercio medio facial; TG'-GN': Profundidad Tercio inferior facial; SN'-PN': Protrusión nasal (Columela), LVVLínea Vertical Verdadera; OR'- Punto orbitario; $\mathrm{CB}^{\prime}$ - Punto hueso malar; $\mathrm{AB}^{\prime}$ - Punto base alar; SP'- Punto subpupilar; PG- Pogonion piel; Angulo NL: naso labial; N: número absoluto; SI : estrato con menor índice de indigenicidad y mayor estrato socioeconómico; SIII: estrato con mayor índice de indigenicidad y menor estrato socioeconómico; X: Valor promedio DS: desviación estándar; M: masculino; F: femenino

Tabla 2 - Significancia estadística al comparar entre sexos en las distintas denticiones para valores que corresponden a Tercio Medio, Tercio Inferior y Desarrollo Nasal

\begin{tabular}{|c|c|c|c|c|c|}
\hline & \multirow[t]{2}{*}{ VARIABLE } & $\begin{array}{c}\text { DENTICIÖN } \\
\text { TEMPORAL } \\
\text { SI }\end{array}$ & $\begin{array}{l}\text { DENTICION } \\
\text { MIXTA } \\
\text { SI }\end{array}$ & $\begin{array}{c}\text { DENTICION } \\
\text { TEMPORAL } \\
\text { SIII }\end{array}$ & $\begin{array}{c}\text { DENTICION } \\
\text { MIXTA } \\
\text { SIII }\end{array}$ \\
\hline & & p & $\mathbf{P}$ & p & p \\
\hline \multirow{5}{*}{$\begin{array}{l}\text { Medidas de } \\
\text { Tercio Medio }\end{array}$} & OR'-LVV & NS & $\mathrm{NS}$ & NS & $P<0,01$ \\
\hline & CB'-LVV & NS & NS & NS & $P<0,05$ \\
\hline & SP'-LVV & NS & NS & NS & $P<0,05$ \\
\hline & $A B^{\prime}-L V V$ & $p<0,05$ & NS & NS & $P<0,05$ \\
\hline & TG'-SN' & NS & NS & NS & NS \\
\hline \multirow{2}{*}{$\begin{array}{l}\text { Medidas de } \\
\text { Tercio Inferior }\end{array}$} & PG'-LVV & NS & NS & NS & NS \\
\hline & TG'-GN' & NS & NS & $\mathrm{NS}$ & NS \\
\hline \multirow{2}{*}{$\begin{array}{l}\text { Medidas de } \\
\text { Desarrollo } \\
\text { Nasal } \\
\end{array}$} & SN'-PN' & NS & NS & NS & NS \\
\hline & Angulo NL & NS & NS & NS & NS \\
\hline
\end{tabular}

Teste de Wilcoxon

TG'-SN': Profundidad Tercio medio facial; TG'-GN': Profundidad Tercio inferior facial; SN'-PN': Protrusión nasal (Columela), LVVLínea Vertical Verdadera; OR'- Punto orbitario; $C^{\prime}$ - Punto hueso malar; AB' $^{\prime}$ - Punto base alar; SP'- Punto subpupilar; PG- Pogonion piel; Angulo NL : naso labial; SI : estrato con menor índice de indigenicidad y mayor estrato socioeconómico; SIII : estrato con mayor índice de indigenicidad y menor estrato socioeconómico; NS : no significativo 
Tabla 3 - Significancia estadística entre estratos según sexo y dentición

\begin{tabular}{|c|c|c|c|c|c|}
\hline & & \multicolumn{2}{|c|}{ HOMBRES } & \multicolumn{2}{|c|}{ MUJERES } \\
\hline & \multirow{2}{*}{$\begin{array}{c}\text { VARIAB } \\
\text { LE }\end{array}$} & $\begin{array}{l}\text { TEMPORAL } \\
\text { SI - SIII }\end{array}$ & $\begin{array}{l}\text { MIXTA } \\
\text { SI - SIII }\end{array}$ & $\begin{array}{l}\text { TEMPORAL } \\
\text { SI - SIII }\end{array}$ & $\begin{array}{l}\text { MIXTA } \\
\text { SI - SIII }\end{array}$ \\
\hline & & $p$ & p & $p$ & $\mathbf{P}$ \\
\hline \multirow{5}{*}{$\begin{array}{l}\text { Medidas de } \\
\text { Tercio Medio }\end{array}$} & OR'-LVV & NS & NS & NS & NS \\
\hline & CB'-LVV & NS & $p<0,05$ & NS & NS \\
\hline & SP'-LVV & NS & NS & NS & NS \\
\hline & $A B^{\prime}-L V V$ & NS & $p<0,05$ & $p<0,01$ & NS \\
\hline & TG'-SN' & NS & NS & NS & $\mathrm{p}<0,05$ \\
\hline \multirow{2}{*}{$\begin{array}{l}\text { Medidas de } \\
\text { Tercio Inferior }\end{array}$} & PG'-LVV & NS & NS & NS & NS \\
\hline & TG'-GN' & NS & NS & NS & NS \\
\hline \multirow{2}{*}{$\begin{array}{l}\text { Medidas de } \\
\text { Desarrollo } \\
\text { Nasal }\end{array}$} & SN'-PN' & $p<0,05$ & $p<0,01$ & $p<0,01$ & $p<0,01$ \\
\hline & $\begin{array}{l}\text { Angulo } \\
\mathrm{NL}\end{array}$ & NS & $p<0,01$ & NS & NS \\
\hline
\end{tabular}

Teste de Wilcoxon

TG'-SN': Profundidad Tercio medio facial; TG'-GN': Profundidad Tercio inferior facial; SN'-PN': Protrusión nasal (Columela), LVVLínea Vertical Verdadera; OR'- Punto orbitario; CB'- Punto hueso malar; AB'- Punto base alar; SP'- Punto subpupilar; PG- Pogonion piel; Angulo NL : naso labial; SI : estrato con menor índice de indigenicidad y mayor estrato socioeconómico; SIII : estrato con mayor índice de indigenicidad y menor estrato socioeconómico; p: probabilidad; NS : no significativo

Tabla 4- Comparaciones entre estratos y Farkas en sexo masculino según dentición

\begin{tabular}{|c|c|c|c|c|c|}
\hline & \multirow{3}{*}{ VARIABLE } & \multicolumn{2}{|c|}{ HOMBRES DENTICION TEMPORAL } & \multicolumn{2}{|c|}{ HOMBRES DENTICION MIXTA } \\
\hline & & SI -FARKAS & SIII -FARKAS & SI -FARKAS & SIII -FARKAS \\
\hline & & $p$ & $\mathbf{P}$ & $p$ & $\mathbf{p}$ \\
\hline $\begin{array}{l}\text { TERCIO } \\
\text { MEDIO }\end{array}$ & TG'-SN' & NS & NS & $p<0,05$ & NS \\
\hline $\begin{array}{l}\text { TERCIO } \\
\text { INFERIOR }\end{array}$ & TG'-GN' & NS & NS & NS & NS \\
\hline $\begin{array}{l}\text { DESARROLLO } \\
\text { NASAL }\end{array}$ & SN'-PN' & $p<0,01$ & NS & NS & $p<0,01$ \\
\hline
\end{tabular}

Teste t-Sudent

TG'-SN': Profundidad Tercio medio facial; TG'-GN': Profundidad Tercio inferior facial; SN'-PN': Protrusión nasal (Columela); SI: estrato con menor índice de indigenicidad y mayor nivel socioeconómico; SIII: estrato con mayor índice de indigenicidad y menor nivel socioeconómico; p: probabilidad; NS: no significativo

Tabla 5 - Comparaciones entre estratos y Farkas en sexo femenino según dentición

\begin{tabular}{|c|c|c|c|c|c|}
\hline & \multirow{3}{*}{ VARIABLE } & \multicolumn{2}{|c|}{ MUJERES DENTICION TEMPORAL } & \multicolumn{2}{|c|}{ MUJERES DENTICION MIXTA } \\
\hline & & SI -FARKAS & SIII -FARKAS & SI -FARKAS & SIII -FARKAS \\
\hline & & p & $\mathbf{P}$ & p & p \\
\hline $\begin{array}{l}\text { TERCIO } \\
\text { MEDIO }\end{array}$ & TG'-SN' & NS & NS & $p<0,01$ & NS \\
\hline $\begin{array}{l}\text { TERCIO } \\
\text { INFERIOR }\end{array}$ & TG'-GN' & NS & NS & $p<0,01$ & $p<0,05$ \\
\hline $\begin{array}{l}\text { DESARROLLO } \\
\text { NASAL }\end{array}$ & SN'-PN' & $p<0,01$ & NS & $p<0,05$ & $p<0,05$ \\
\hline
\end{tabular}

Teste t-Sudent

TG'-SN': Profundidad Tercio medio facial; TG'-GN': Profundidad Tercio inferior facial; SN'-PN': Protrusión nasal (Columela); SI: estrato con menor índice de indigenicidad y mayor nivel socioeconómico; SIII: estrato con mayor índice de indigenicidad y menor nivel socioeconómico; p: probabilidad; NS: no significativo

\section{DISCUSIÓN}

Por todos es conocida la dificultad de aplicar parámetros internacionales en poblaciones específicas, mientras no hayan sido comprobados como posibles de aplicar según las distintas caracterís- ticas étnicas, estructurales y de miscegenación.

En el caso de Latinoamérica, generalmente se trabaja con parámetros americanos como referencia, aplicando sus estándares al diagnostico y tratamiento de pacientes. En el caso de poblaciones Chilenas, no existen estudios que comparen estas mediciones, por lo que los 
resultados del presente trabajo serán un aporte a la clinica fonoaudiológica, en cuanto a la caracterización de los pacientes y su posterior tratamiento, basado en evidencias inherentes a su población.

Al comparar los resultados según sexo, no se encontraron diferencias significativas, sin embargo, se observa una tendencia en los hombres hacia una mayor distancia anteroposterior con respecto a la LVV. Esta tendencia se acentúa en dentición mixta, lo cual puede explicarse por la evolución del crecimiento y desarrollo corporal. Esto se correspondería con lo descrito por Arnett ${ }^{19}$ en población adulta caucásica, por lo cual se podría pensar que esta tendencia se comienza a manifestar durante la niñez y se establece claramente en población adulta. Coincidente con lo descrito por otros autores como McNamara ${ }^{28}$ y otros, no se encontraron diferencias entre sexos para las medidas de ángulo nasolabial, protrusión nasal, profundidad de tercio medio y profundidad de tercio inferior, dichos autores señalan que no debieran existir deferencias entre sexos antes de llegar al pick puberal, donde las diferencias comenzarían a ser más significativas ${ }^{28,29}$.

En cuanto a la comparación por estrato, al comparar el grupo de medidas anteroposteriores del tercio medio con respecto a la LVV en la totalidad de la muestra, no se observan diferencias consistentes atribuibles a diferencias étnicas ni de desarrollo. Las diferencias puntuales, especialmente en punto base alar (AB'-LVV) son atribuibles a factores de procedimiento como lo describe Farkas ${ }^{27}$, quien señala que el punto base alar es difícil de visualizar desde una vista lateral, ya que el grosor de la mejilla puede enmascarar su posición real. La tendencia del grupo sociogenético SI a tener un ángulo nasolabial más abierto, asociado a la tendencia de mayores valores de protrusión nasal, indicaría que el grupo SI presenta un mayor desarrollo nasal. Estos resultados podrían estar indicando una influencia genética que provoca diferencias morfológicas nasales.

En profundidad de tercio medio e inferior entre ambos estratos sociogenéticos, para ambos sexos, no se encuentran diferencias que reflejen una variabilidad en el crecimiento.

Los valores obtenidos este estudio con respecto a las medidas analizadas por Farkas en su investigación, son bastantes similares, a pesar que se expresan algunas diferencias significativas, éstas pueden ser atribuidas a una diferencia de tamaño de muestra y a la diferencia de metodología, ya que en este trabajo el análisis fue fotográfico ${ }^{27}$.

Considerando que la estética facial es un aspecto que trabajan los fonoaudiólogos que se dedican al área de motricidad orofacial y los odontólogos, resulta interesante contar con esta información referida a poblaciones mixtas, es decir que presentan algún grado de mezcla étnica, de tal forma de utilizar los parámetros antes mencionados como referencias validas, tanto para la evaluación como para el tratamiento de los pacientes, especialmente los que requieren un tratamiento conjunto.

La percepción de la estética facial es subjetiva, cambia en el tiempo y de población en población, por lo que es necesario continuar investigando y discutiendo las bases filosóficas, mientras exista un desafió tratar a los pacientes que requieren un cambio facial ${ }^{5}$.

\section{CONCLUSIONES}

No existen diferencias entre sexos para las medidas estudiadas. No se encuentran diferencias por estrato sociogenético en las medidas de profundidad de tercio medio e inferior, ni tampoco en el grupo de medidas anteroposteriores con respecto a la línea Vertical Verdadera.

Se encuentra un mayor desarrollo nasal en el estrato con menor índice de indigenicidad y mayor estrato socioeconómico, que en el estrato con mayor índice de indigenicidad y menor estrato socioeconómico, lo que se ve reflejado en mayores valores de protrusión nasal y ángulo nasolabial.

Los resultados de las comparaciones con las medidas en niños norteamericanos de Farkas no permiten establecer diferencias entre poblaciones.

\begin{abstract}
Purpose: to compare anthropometric measurements of facial esthetics in 5 and 8 years old children, with normal physical and occlusal characteristics, belonging to different sociogenetic groups from Santiago de Chile (mixed ethnic population); and with north American Caucasian parameters published by Farkas. Methods: a facial analysis based on Arnett and Farkas was carried out, using digital photography supported by a clinical examination, in four samples, according to the dentition and sociogenetic stratum. Results: the results show no differences in Chile according to sex and stratum, neither with Caucasian measurements. Conclusion: the anthropometric measurements of facial aesthetics in sociogenetic groups from Santiago are not different with Farkas Caucasian measurements and can be used for clinical purposes in mixed ethnically Chilean populations.
\end{abstract}

KEYWORDS: Ethnic Groups; Esthetics; Anthropometry 


\section{REFERENCIAS}

1.Salinas ER, Carvajal A, Azofeifa J, Urrutia $\mathrm{H}$. Relación entre perfil blando y perfil óseodentario subyacente. Rev Chil Ortodoncia. 1990; 7:78-89.

2.Monsalve C, Guerrero S. Estudio del patrón facial del joven chileno. Rev Odontol Chil. 2003; 51(1):39-45.

3. Farkas LG, Katic MJ, Forrest CR, Alt KW, Bagic I, Baltadjiev G, et al. International anthropometric study of facial morphology in various ethnic groups/races. J Craniofac Surg. 2005; 16(4):615-46.

4. Arnett GW, Bergman RT. Facial keys to orthodontic diagnosis and treatment planning. Part I. Am J Orthod Dentofacial Orthop. 1993; 103(4):299-312.

5.Naini FB, Moss JP, Gill DS. The enigma of facial beauty: esthetics, proportions, deformity, and controversy. Am J Orthod Dentofacial Orthop. 2006; 130(3):277-82.

6. Fernández-Riveiro $P$, Smyth-Chamosa E, Suárez-Quintanilla D, Suárez-Cunqueiro M. Angular photogrammetric analysis of the soft tissue facial profile. Eur J Orthod. 2003; 25(4):393-9.

7. Nunes P. Fonoaudiologia \& estética: a motricidade orofacial aplicada na estética da face. 1. ed. São Paulo: Lovise; 2006. 136 p.

8. Amaral EC, Bacha SMC, Ghersel ELA, Rodrigues PMI. Inter-relação entre a odontologia e a fonoaudiologia na motricidade orofacial. Rev CEFAC. 2006; 8(3):337-51.

9. Marchesan IQ, Sanseverino NT. Fonoaudiologia y ortodoncia/ortopedia facial. São José dos Campos: Pulso; 2004.

10. Edler R, Agarwal P, Wertheim D, Greenhill $D$. The use of anthropometric proportion indices in the measurement of facial attractiveness. Eur J Orthod. 2006; 28(3):274-81.

11. Cattoni DM, Miranda FD, Marchesan IQ, Latorre MRDPO. Medidas antropométricas faciais em crianças segundo períodos da dentição mista. Rev CEFAC. 2003; 5(1):21-9.

12. Medeiros F, Toledo MR, Gomes ICD, Marchesan IQ. Diferenças antropométricas entre mulheres brancas e negras após crescimento puberal.

Rev CEFAC. 2005; 7(4):459-65.

13. Farkas LG, Eiben OG, Sivkov S, Tompson $B$, Katic MJ, Forrest CR. Anthropometric measurements of the facial framework in adulthood: age-related changes in eight age categories in 600 healthy white North Americans of European ancestry from 16 to 90 years of age. J Craniofac Surg. 2004 15(2):288-98.
14. Farkas LG, Hreczko TM, Katic MJ, Forrest $\mathrm{CR}$. Proportion indices in the craniofacial regions of 284 healthy North American white children between 1 and 5 years of age. J Craniofac Surg. 2003; 14(1):13-28.

15. Farkas LG, Posnick JC. Growth and development of regional units in the head and face based on anthropometric measurements. Cleft Palate Craniofac J. 1992; 29(4):301-2.

16.Proffit W, Fields H. Ortodoncia contemporánea: teoría y práctica. 2. ed. España: Mosby-Doyma Libros; 1994. p. 143-54.

17.Farkas LG, Posnick JC, Hresczko TM. Growth patterns of the face: a morphometric study. Cleft Palate Craniofac J. 1992; 29(4):308-15.

18. Arnett GW, Bergman RT. Facial keys to orthodontic diagnosis and treatment planning. Part II. Am J Orthod Dentofacial Orthop. 1993; 103(5):395-411.

19. Arnett GW, Jelic JS, Kim J, Cummings DR, Beress A, Worley Junior CM, et al. Soft tissue cephalometric analysis: diagnosis and treatment planning of dentofacial deformity. Am J Orthod Dentofacial Orthop. 1999; 116(3):239-53.

20. Valenzuela C, Acuña M, Harb Z. Gradiente sociogenética en la población chilena. Rev Med Chile. 1987; 115:295-9.

21. Valenzuela C. Marco de referencia sociogenético para los estudios de salud pública en Chile. Rev Chil Pediatr. 1984; 55(2):123-7.

22. Palomino HM, Palomino H, Cauvi D, Barton SA, Chakraborty R. Facial clefting and amerindian admixture in population of Santiago, Chile. Am J Hum Biol. 1997; 9:225-32.

23. Goycoolea A, Palomino HM, Palomino H, Blanco R. Agentes medioambientales en la susceptibilidad a las fisuras faciales en el norte de Chile. Odontol Chil. 1993; 41:113-9.

24. Olivares S, Zacarías I, Burrows R, Díaz E, Urbina R. Guía de alimentación del escolar de 6 a 10 años. Universidad de Chile, Instituto de Nutrición y Tecnología de los Alimentos y Gobierno de Chile. Disponível em: URL: http://www.inta.cl/consumidor/niños/ index.asp?offset $=1$

25. Espinoza R, Cauvi D, Miranda T, Urzúa S, Alvarez F, Palacios $S$, et al. Texto de autoenseñanza: evolución de la dentición. v. 1. Chile: Ediciones de la Facultad de Odontología; 1996. p. 118.

26. Sprandley FL, Jacobs JD, Crowe DP. Assessment of the anteroposterior soft-tissue contour of the lower facial third in the ideal young adult. Am J Orthod. 1981; 79(3):316-25.

27. Farkas LG, editor. Anthropometry of the head and face. 2. ed. New York: Raven Press. 1994. p. 3-57, p. 79-89. 
28. Ursi WJ, Trotman CA, McNamara Junior JA, Behrents RG. Sexual dimorphism in normal craniofacial growth. Angle Orthod. 1993;63(1):47-56.
29. Nanda RS, Meng H, Kapila S, Goorhuis J. Growth changes in the soft tissue facial profile. Angle Orthod. 1990; 60(3):177-90.

RECEBIDO EM: 08/08/06

ACEITO EM: 13/11/06

Endereço para correspondencia:

Departamento del Niño y Ortopedia Dentomaxilar

Facultad de Odontología, U de Chile.

Santa María 571-Recoleta

Santiago, Chile

Tel: 5629785027

E-mail: illanpalom@manquehue.net 